\title{
Expression of metallothionein I/II and Ki-67 antigen in various histological types of basal cell carcinoma
}

\author{
Andrzej Bieniek ${ }^{1}$, Bartosz Pula ${ }^{2}$, Aleksandra Piotrowska ${ }^{2}$, \\ Marzena Podhorska-Okolow ${ }^{2}$, Anna Salwa ${ }^{1}$, Maria Koziol' ${ }^{1}$, Piotr Dziegiel ${ }^{2,3,4}$ \\ ${ }^{1}$ Department of Dermatology, Venerology and Allergology, Medical University, Wroclaw, Poland \\ ${ }^{2}$ Department of Histology and Embryology, Medical University, Wroclaw, Poland \\ ${ }^{3}$ Lower Silesia Center of Oncology, Wroclaw, Poland \\ ${ }^{4}$ Department of Histology and Embryology, Poznan University of Medical Sciences, Poznan, Poland
}

\begin{abstract}
Basal cell carcinoma (BCC) is the most frequent skin cancer, with many different histological subtypes. Recent studies have investigated the expression of proliferative markers, but little is known about the expression of metallothioneins (MT) in different histological subtypes of this cancer and their impact on proliferation intensity in BCC. In this study, we examined MT-I/II expression by immunohistochemistry in 58 different histological subtypes of BCC (38 nodular, six adenoid, eight infiltrative, and six metatypic cases) and correlated its expression with tumor size and Ki-67 proliferation rate. Statistical analysis revealed no significant differences in the expression of studied markers in regard to the histological subtype. A positive correlation between MT and Ki-67 expression was observed for all the studied cases $(r=0.26 ; p=0.049)$, but was even stronger in the metatypic subtype of BCC $(r=0.85 ; \mathrm{p}=0.033)$. MT and Ki-67 expression did not correlate with tumor size. In conclusion, it seems that metallothioneins may have an impact on the proliferation rate of BCC, but further studies are required to determine whether MT may be a risk factor of recurrences. (Folia Histochemica et Cytobiologica 2012, Vol. 50, No. 3, 352-357)
\end{abstract}

Key words: metallothionein, Ki-67, BCC

\section{Introduction}

Basal cell carcinoma (BCC, carcinoma basocellulare) represents a non-uniform group of tumors which are characterized by slow progression, and the very rare (below $0.1 \%$ of cases) development of metastases [1]. BCC is the most frequent invasive skin tumor in Europe, comprising around $80 \%$ of non-melanoma skin cancers [2]. In most cases, it involves slowly growing tumors of a relatively low aggressiveness. A small minority of BCC leads to relapses and local infiltration of tissue structures which may lead to death of the host [2].

Correspondence address: P. Dziegiel,

Department of Histology and Embryology,

Medical University in Wroclaw,

Chalubinskiego Str. 6a, 50-368 Wroclaw, Poland;

tel.: + 487178413 54, fax: + 487178400 82;

e-mail: piotr.dziegiel@hist.am.wroc.pl
Depending on histological and clinical traits, more than ten forms of the tumor can be distinguished [3]. The isolation of individual types of $\mathrm{BCC}$ is useful due to the variable character of its development (variable aggressiveness), which determines the selection of an appropriate therapeutic approach $[4,5]$. The nodular and superficial forms manifest no particular aggressiveness and can often be cured. The varieties manifesting higher aggressiveness and more frequent recurrences include micronodular, morpheaform, infiltrative and metatypic types. They may aggressively infiltrate and destroy surrounding tissues, and in specific conditions may endanger the patient's life [6]. The superficial type manifests no particular aggressiveness but frequently shows recurrences [5]. Due to the frequent coexistence of various forms of BCC within a single lesion and, occasionally, unpredictable behavior of the tumor, the identification of markers which are independent of histopathological structure 
Table 1. Patient and tumor characteristics

\begin{tabular}{|l|c|c|c|}
\hline & No. of cases (\%) & $\begin{array}{c}\text { Age (years) } \\
\text { Mean (range) }\end{array}$ & $\begin{array}{c}\text { Tumour diameter [mm] } \\
\text { Mean (range) }\end{array}$ \\
\hline BCC & $58(100)$ & $65.98(21-87)$ & $20.63(8-70)$ \\
\hline Adenoid BCC & $6(10.3)$ & $62.17(52-72)$ & $21.33(15-30)$ \\
\hline Infiltrative BCC & $8(13.9)$ & $64.13(47-81)$ & $21.71(12-35)^{*}$ \\
\hline Metatypic BCC & $6(10.3)$ & $71.67(45-85)$ & $30.00(10-70)$ \\
\hline Nodular BCC & $38(65.5)$ & $66.08(21-87)$ & $18.84(8-50)$ \\
\hline
\end{tabular}

*Data missing for one case

but would be helpful in establishing a prognosis, is urgently needed $[2,5]$.

Expression of metallothionein (MT) in tumor cells represents a relatively new prognostic index in various neoplastic diseases, as indicated by numerous studies. MT is a low molecular weight protein (around $7 \mathrm{kDa}$ ) which is widely expressed in cells (nuclei and/ /or cytoplasm) of various organs and tumors $[6,7]$. Depending on the type, MT consists of 61-68 amino acids, forming two chains of $\alpha$ and $\beta$, linked by a lysine dimer. Considering their structure and manifestation, four basic types of MT can be distinguished, including MT-I, MT-II, MT-III, MT-IV [6, 7]. Binding of heavy metal ions, either indispensable for MT function or toxic, represents a principal character of MT. The protein plays a protective role, preventing against intoxication with heavy metals, such as $\mathrm{Pb}, \mathrm{Hg}, \mathrm{Cu}$, $\mathrm{Cd}[8,9]$. MT manifests also a strong anti-oxidative activity, protecting cells from the damaging effects of reactive oxygen species, ionizing radiation and chemotherapeutic agents [10-12]. Intensely dividing cells (including tumor cells) manifest an increased expression of MT, which supplies zinc (Zn) ions for enzymes involved in DNA replication and protects cells from apoptosis [13]. This has induced investigators to examine the expression of MT in various tumors [14-16]. An increase in MT expression in tumor cells correlates with expression of $\mathrm{Ki}-67$ proliferation antigen and a less favorable outcome of the disease $[6,7,14-$ -16]. It has been shown that MT-2A isoform expression in breast cancer cells stimulates cell proliferation, but does not exert anti-apoptotic properties [17]. Moreover, a decline of MT-I/II expression is associated with a decrease of PCNA and Ki-67 expression both in sun-exposed and sun-protected skin, which may indicate its impact on cell proliferation [18]. It has been suggested that MT-I/II may protect skin tissues from ultraviolet radiation (UVR) induced carcinogenesis on the one hand, but may induce tumor growth once these occur [19].

Until now, only individual studies have been published in which MT expression was studied in skin cancers [20, 21]. The studies showed an increased expression of MT in spinocellular carcinoma and a less typical increased expression of MT in aggressive varieties of $\mathrm{BCC}$, but none of the investigations dealt with the relationship between expressions of MT and Ki-67 antigen in various histological types of BCC.

Our study aimed at determining the intensity of MT-I/II expressions using the immunohistochemical method in various histological types of BCC, and correlating its expressions with the expression intensity of Ki-67 antigen and tumor size.

\section{Material and methods}

Patients. Studies were performed on the material of various histological types of basal cell carcinomas ( 58 cases) obtained during excision of the tumor in the Department of Dermatology, Venerology and Allergology, Medical University in Wroclaw between 2005 and 2007. The clinical and pathological data are summarized in Table 1 . The study was approved by the Commission of Bioethics at Wroclaw Medical University.

Immunohistochemistry. The tumor samples were fixed in $10 \%$ buffered formalin, dehydrated and embedded in paraffin blocks, of which hematoxylin and eosin (HE) stained slides were made and used for verification of the histopathological diagnosis. All immunohistochemical reactions (IHC) were performed on 4- $\mu \mathrm{m}$-thick paraffin sections mounted on Superfrost Plus slides (Menzel Gläser, Braunschweig, Germany). First, sections were deparaffinized in xylene and rehydrated. In the case of anti-Ki-67 IHC reactions, the sections were incubated in a citrate buffer $\left(\mathrm{pH} 6,10 \mathrm{mM} ; 95^{\circ} \mathrm{C}\right.$, $20 \mathrm{~min}$ ) in order to retrieve the antigens. Activity of endogenous peroxidase was blocked by $5 \mathrm{~min}$ incubation with $3 \%$ solution of $\mathrm{H}_{2} \mathrm{O}_{2}$. Expression of MT-I/II and Ki-67 antigen was demonstrated using mouse monoclonal anti-MT-I/II (clone E9, dilution 1:100) and anti-Ki-67 (clone MIB-1, dilution 1:100) antibodies, respectively. Sections were incubated with primary antibodies overnight at $4^{\circ} \mathrm{C}$. The studied antigens were visualized using biotinylated antibodies and streptavidin, conjugated with horseradish peroxidase 
(LSAB+ System-HRP). Diaminobenzidine (DAB) served as a substrate. The reactions were accompanied by a negative control. Subsequently, the preparations were counterstained with hematoxylin. All the antibodies and reagents originated from DakoCytomation (Glostrup, Denmark).

Evaluation of IHC reactions. The intensity of MT expression in tumor cells was evaluated using the semi-quantitative technique of Remmele and Stegner [22]. In every case, the semi-quantitative immunoreactive score (IRS) of IHC was calculated by multiplying the grade of scale defining proportion of positive cells $(0-4)$ by the degree of scale defining intensity of color reaction (1-3), yielding a final score of 0 to 12. Intensity of Ki-67 antigen expression in tumor cells was evaluated by the proportion of positive cells among tumor cells as follows: 0 pts - no reaction; $1 \mathrm{pt}-1-10 \% ; 2 \mathrm{pts}-$ $11-25 \%$; 3 pts $-26-50 \% ; 4$ pts $->50 \%$ positive cells.

Statistical analysis. The obtained results were subjected to statistical analysis using Prism 5.0 software (GraphPad, CA, USA). Shapiro-Wilk test was applied to estimate the normality of distribution. Spearman's correlation test, the Kruskal-Wallis test, and the U test of Mann and Whitney were used to compare the results. Differences were accepted to be significant at $\mathrm{p}<0.05$.

\section{Results}

Cytoplasmatic/nuclear expression of MT and nuclear expression of $\mathrm{Ki}-67$ antigen were detected in all examined cases of the studied basal cell carcinomas (Figure 1). The highest intensity of MT-I/II expression was noted in adenoid BCC $(5.17 \pm 2.93)$, and the lowest in metatypic BCC $(4.33 \pm 1.97)$. The most pronounced expression of $\mathrm{Ki}-67$ antigen was detected in the metatypic type of BCC $(2.66 \pm 1.03)$, and the lowest intense one in nodular BCC $(1.90 \pm 0.89)$. Expression intensities of the studied antigens for all the examined cases and individual histopathological types are presented in Table 2. No significant differences were detected in MT-I/II expression or Ki-67 expression between individual histological types of BCC.

A positive correlation was demonstrated between expression intensities of Ki-67 antigen and MT-I/II, when the analysis took into account all 58 studied cases of $\mathrm{BCC}(\mathrm{r}=0.26 ; \mathrm{p}=0.049$; Figure 2A). Among the studied subtypes, only the metatypic BCC subtype demonstrated a strong positive correlation between the two studied markers (MT-I/II and Ki-67) $(r=0.85 ; p=0.033 ;$ Figure 2B). In cases of the other studied subtypes, no significant correlation was observed between the studied markers.

Neither in the entire studied group, nor within individual histopathological types, could any relation-
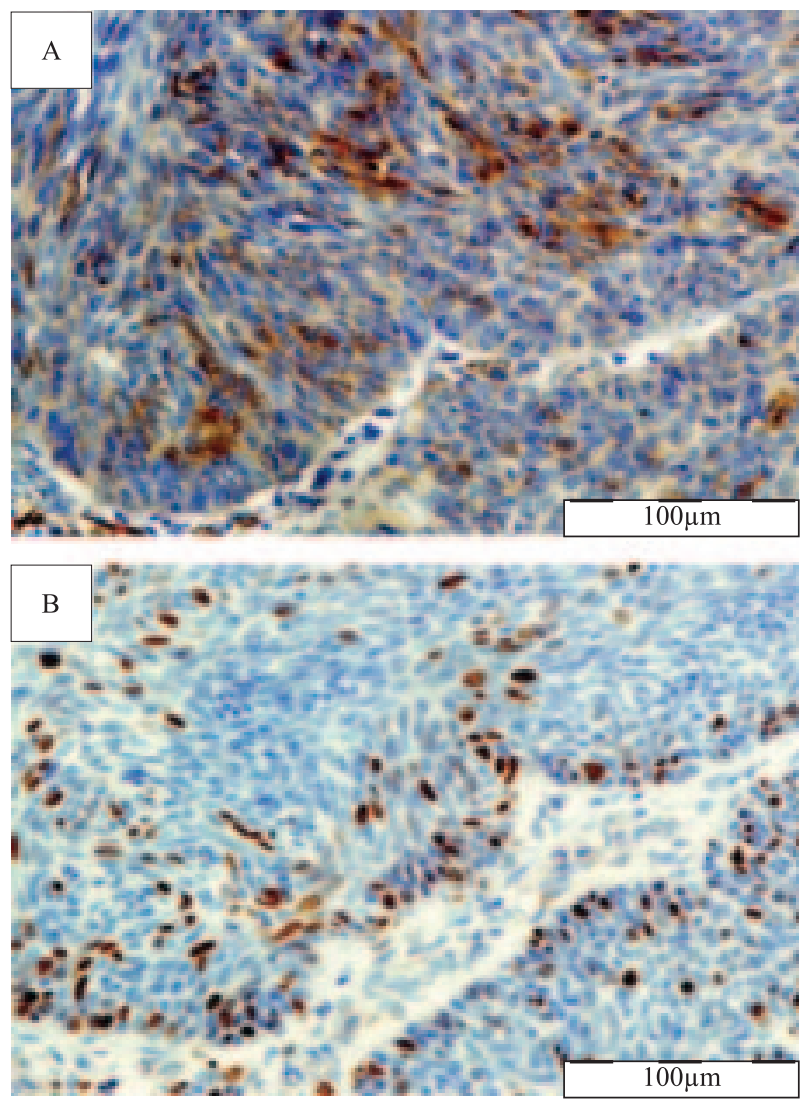

Figure 1. Cytoplasmatic/nuclear expression of MT-I/II (A) and nuclear expression of Ki-67 antigen (B) in cells of BCC

Table 2. Expression of Ki-67 antigen and MT I/II in histological subtypes of BCC

\begin{tabular}{|l|c|c|}
\hline & $\begin{array}{c}\text { Ki-67 expression } \\
(\text { Mean } \pm \text { SD) }\end{array}$ & $\begin{array}{c}\text { MT-I/II expression } \\
(\text { Mean } \pm \text { SD })\end{array}$ \\
\hline BCC & $2.02 \pm 0.93$ & $4.50 \pm 2.44$ \\
\hline Adenoid BCC & $2.17 \pm 0.98$ & $5.17 \pm 2.93$ \\
\hline Infiltrative BCC & $2.00 \pm 0.93$ & $4.63 \pm 1.60$ \\
\hline Metatypic BCC & $2.67 \pm 1.03$ & $4.33 \pm 1.97$ \\
\hline Nodular BCC & $1.90 \pm 0.89$ & $4.40 \pm 2.64$ \\
\hline
\end{tabular}

ship be detected between intensity of Ki-67 antigen or MT-I/II expression on the one hand and diameter of the tumor on the other.

\section{Discussion}

Since the mid-1990s, numerous studies have been published related to expression of various antigens, using e.g. IHC in skin carcinomas (mainly basal cell carcinomas and spinocellular carcinomas). In these studies, the authors have suggested that a variable expression of certain antigens in tumor cells (in the cell nucleus or in the cytoplasm) or in cells of their sublay- 

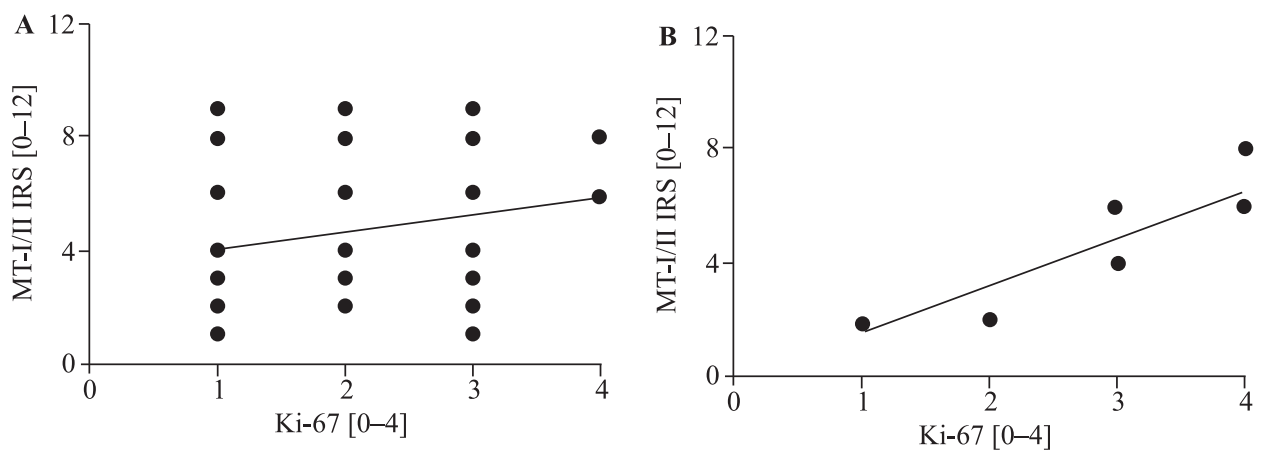

Figure 2. Correlation between expression of MT-I/II and Ki-67 antigen in all the analyzed samples (A) and metatypic BCC (B)

er may correlate with the aggressiveness of the tumors, providing important prognostic indices. Potential advantages have also been suggested of using IHC studies for easier differentiation between individual varieties of BCC as well as between BCC and tumors of a similar histomorphology (e.g. trichoepithelioma) [21, 23]. Results obtained in IHC studies conducted on these types of tumors have frequently been equivocal.

Ionesco et al. performed a comparative analysis of $\mathrm{Ki}-67, \mathrm{p} 53$ and $\mathrm{Bcl}-2$ antigen expression in two groups: the more aggressive (metastatic) BCC tumors and $\mathrm{BCC}$ of a benign course. They demonstrated no significant differences between the groups in expression of the antigens [24]. On the other hand, Yerbakan et al. examined expression of Ki-67, CD31 and epidermal growth factor receptor (EGFR) in groups of $\mathrm{BCC}$ of variable aggressiveness. They found that expression intensity of the mentioned antigens was significantly higher in recurrent (i.e. more aggressive) tumors, compared to primary tumors [25]. In a similar way, elevation in Ki-67 expression level was detected by Healy et al. in BCC tumors which relapsed after treatment, compared to tumors which were cured [26]. The cellular proliferation characteristics of various histological/clinical types of BCC were analyzed by Horlock et al. using analysis of Ki-67 antigen expression [27]. Their investigations showed that intensity of proliferation measured by the level of $\mathrm{Ki}-67$ antigen expression was lower in the nodular and micronodular types, and higher in scleroderma-like, infiltrating and superficial BCC types, which is consistent with our observations. According to the authors, a correlation can be noted between higher expression of Ki-67 antigen and BCC variations of higher aggressiveness [27]. However, the authors seemed to pay no attention to the significant aggressiveness manifested by the micronodular type of BCC, manifesting in their hands low levels of Ki-67 antigen expression $[4,27]$. Other proliferation markers, including PCNA and $\mathrm{Ki}-67$ in various types of BCC, were also analyzed by Barrett et al. [28]. In this study, nu- clear reaction of PCNA expression demonstrated higher intensity than expression of Ki-67. PCNA was manifested in less than $10 \%$ of BCC cells in the nodular form, in more than $30 \%$ of cells in most tumors in the scleroderma-like and infiltrating forms, and in all tumors of the metatypic form [28]. Another study which compared intensity of Ki-67 antigen expression to depth of infiltration and histological type of BCC demonstrated no relationship between the parameters [29].

The results of the above-mentioned studies are consistent with the results obtained by us showing no differences between histological types of BCC and tumor size. Janisson-Dargaud et al. also demonstrated no differences in the expression of proliferation markers between recurrent and non-recurrent forms, and only the frequency of aneuploidy proved to represent a prognostic factor linked to the risk of recurrence [30].

Rossen et al. analyzed levels of expression of MT-I/II isoforms in BCC compared to normal epidermis and benign epidermal hypertrophy, including basaloidal hypertrophy, covering solid fibromas [20]. Within normal epidermis, the expression of MT-I/II was documented in cytoplasm of basal cells. A similar form of MT expression was demonstrated in a hypertrophic epidermis. Basaloidal proliferations, as well as superficial and nodular forms of BCC, demonstrated a decreased MT-I/II expression (in approximately $92 \%$ of the cases) [20]. Nevertheless, in $86 \%$ of cases of infiltrating BCC, increased expression of MT-I/II was demonstrated in neoplastic cells [20]. Our results seem to confirm this observation. The increased expression of MT-I/II in infiltrating BCC type may be linked to an increase in clinical malignancy. Interestingly, the metatypic BCC showed comparable low MT-I/II expression with the nodular BCC subtype, although the clinical course of the latter is more benign. Moreover, in the metatypic BCC, a weak correlation between MT-I/II and Ki-67 antigen expression was noted, which may 
point to more pronounced pro-proliferative effects of MT-I/II expression in this particular type. Although metatypic $\mathrm{BCC}$ is regarded as an aggressive subtype of BCC, it manifested the lowest MT-I/II expression of the analyzed subtypes. We used IHC techniques to determine MT-I/II expression, but recent studies have shown that only particular MT-I/II isoforms may contribute to cancer cell aggressiveness in some tumor types [31-35]. In our study, we noted the highest Ki-67 antigen expression in this subtype. Other investigations related to the expression of MT in BCC, SCC and healthy skin have also demonstrated an increased level of MT-I/II expression in neoplastic tissues, which may point to the role of MT-I/II in the progression of basal cell carcinoma [21].

Even if our studies have failed to document significant differences in the expression of Ki-67 antigen and MT-I/II expression between various histological types of BCC, its nodular type, manifesting the most benign clinical course, has shown the lowest level of Ki-67 antigen expression and a comparable expression of MT-I/II compared to the other histological types. The positive correlation between expression of $\mathrm{Ki}-67$ and MT-I/II is worth accentuating, as it confirms the role of MT-I/II in the proliferation of neoplastic cells $[6,7,13]$. Moreover, in the metatypic variety of $\mathrm{BCC}$, the correlation coefficient has proven to be very high despite the low number of examined cases, which may indicate a significant effect of MT-I/II on the proliferation process in this histological type of BCC. In this study, MT-I/II and Ki-67 antigen expression did not correlate with tumor size, and the absence of unequivocal data related to the risk of recurrence does not permit the recognition of Ki-67 antigen or MT-I/II as prognostic factors in BCC, but does give new insights into the biology of BCC. However, our study was limited by the small number of particular cases of BCC subtypes. Therefore, our findings cannot be generalized. Further studies are necessary to confirm the role of MT-I/II in different BCC subtypes.

\section{Acknowledgments}

This study was supported by a scientific grant from Wroclaw Medical University, No. 1507.

\section{References}

1. Marks R. The epidemiology of non-melanoma skin cancer: who, why and what can we do about it. $J$ Dermatol. 1995;22:853-857.

2. Rubin AI, Chen EH, Ratner D. Basal-cell carcinoma. NEngl J Med. 2005;353:2262-2269.

3. Wade TR, Ackerman AB. The many faces of basal-cell carcinoma. J Dermatol Surg Oncol. 1978;4:23-28.
4. Crowson AN. Basal cell carcinoma: biology, morphology and clinical implications. Mod Pathol. 2006;19(Suppl 2):127-147.

5. Mosterd K, Arits AH, Thissen MR et al. Histology-based treatment of basal cell carcinoma. Acta Derm Venereol. 2009;89:454-458.

6. Dziegiel P. Expression of metallothioneins in tumor cells. Pol J Pathol. 2004;55:3-12.

7. Pedersen MǨ, Larsen A, Stoltenberg M et al. The role of metallothionein in oncogenesis and cancer prognosis. Prog Histochem Cytochem. 2009;44:29-64.

8. Braun W, Vas $\alpha$ k M, Robbins AH et al. Comparison of the NMR solution structure and the x-ray crystal structure of rat metallothionein-2. Proc Natl Acad Sci USA. 1992;89:10124-10128.

9. Schultze P, Wörgötter E, Braun W et al. Conformation of [Cd7]-metallothionein-2 from rat liver in aqueous solution determined by nuclear magnetic resonance spectroscopy. J Mol Biol. 1988;203:251-268.

10. Koropatnick J, Kloth DM, Kadhim S et al. Metallothionein expression and resistance to cisplatin in a human germ cell tumor cell line. J Pharmacol Exp Ther. 1995;275:1681-1687.

11. Satoh M, Cherian MG, Imura N et al. Modulation of resistance to anticancer drugs by inhibition of metallothionein synthesis. Cancer Res. 1994;54:5255-5257.

12. Lohrer H, Robson T. Overexpression of metallothionein in $\mathrm{CHO}$ cells and its effect on cell killing by ionizing radiation and alkylating agents. Carcinogenesis. 1989;10:2279-2284.

13. Cherian MG, Apostolova MD. Nuclear localization of metallothionein during cell proliferation and differentiation. Cell Mol Biol. 2000;46:347-356.

14. Dziegiel P, Forgacz J, Suder E et al. Prognostic significance of metallothionein expression in correlation with Ki-67 expression in adenocarcinomas of large intestine. Histol Histopathol. 2003;18:401-407.

15. Dziegiel P, Suder E, Surowiak P et al. Expression of metallothionein in synovial sarcoma cells. Appl Immunohistochem Mol Morphol. 2002;10:357-363.

16. Dziegiel P, Salwa-Żurawska W, Żurawski J et al. Prognostic significance of augmented metallothionein (MT) expression correlated with $\mathrm{Ki}-67$ antigen expression in selected soft tissue sarcomas. Histol and Histopathol. 2005;20:83-89.

17. Jin R, Chow VT, Tan PH et al. Metallothionein 2A expression is associated with cell proliferation in breast cancer. $\mathrm{Car}$ cinogenesis. 2002; 23:81-86.

18. Ma C, Li LF, Chen X. Expression of metallothionein-I and II in skin ageing and its association with skin proliferation. $\mathrm{Br}$ J Dermatol. 2011;164:479-482

19. McGee HM, Woods GM, Bennett B et al. The two faces of metallothionein in carcinogenesis: photoprotection against UVR-induced cancer and promotion of tumour survival. Photochem Photobiol Sci. 2010;9:586-596.

20. Rossen K, Haerslev T, Hou-Jensen K et al. Metallothionein expression in basaloid proliferations overlying dermatofibromas and in basal cell carcinomas. Br J Dermatol. 1997;136:30-34.

21. Borges Pr, Ribeiro R., Cardoso SV et al. Metallothionein Immunolocalization in Actinic Skin Nonmelanoma Carcinomas. Appl Immunohistochem Mol Morphol. 2007;15:165-169.

22. Remmele W, Stegner HE. Recommendation for uniform definition of an immunoreactive score (IRS) for immunohistochemical estrogen receptor detection (ER-ICA) in breast cancer tissue. Pathologe. 1987;8:138-140.

23. Abdelsayed RA, Guijarro-Rojas M, Ibrahim NA et al. Immunohistochemical evaluation of basal cell carcinoma and trichepithelioma using Bcl-2, Ki67, PCNA and P53. J Cutan Pathol. 2000;27:169-175. 
24. Ionescu DN, Arida M, Jukic DM. Metastatic Basal Cell Carcinoma - Four Case Reports, Review of Literature, and Immunohistochemical Evaluation. Arch Pathol Lab Med. 2006;130:45-51.

25. Yerebakan O, Ciftcioglu MA, Akkaya BK et al. Prognostic value of $\mathrm{Ki}-67, \mathrm{CD} 31$ and epidermal growth factor receptor expression in basal cell carcinoma. J Dermatol. 2003;30:33-41 .

26. Healy E, Angus B, Lawrence CM, Rees JL et al. Prognostic value of Ki67 antigen expression in basal cell carcinomas. $\mathrm{Br}$ J Dermatol. 1995;133:737-741.

27. Horlock NM, Wilson GD, Daley FM et al. Cellular proliferation characteristics of basal cell carcinoma: relationship to clinical subtype and histopathology. Eur J Surg Oncol. 1997;23:247-252.

28. Barrett TL, Smith KJ, Hodge JJ et al. Immunohistochemical nuclear staining for p53, PCNA, and Ki-67 in different histologic variants of basal cell carcinoma. Dermatopathology. 1997:37:430-437

29. Son KD, Kim TJ, Lee YS et al. Comparative Analysis of Immunohistochemical Markers With Invasiveness and Histologic Differentiation in Squamous Cell Carcinoma and Basal Cell Carcinoma of the Skin. J Surg Oncol. 2008;97:615-620.
30. Janisson-Dargaud D, Durlach A, Lorenzato M et al. Aneuploidy, but not Ki-67 or EGFR expression, is associated with recurrences in basal cell carcinoma. J Cutan Pathol. 2008;35:916-921.

31. Tai SK, Tan OJ, Chow VT et al. Differential Expression of Metallothionein 1 and 2 Isoform in Breast Cancer Lines with Different Invasive Potential Identification of a Novel Nonsilent Metallothionein-1H Mutant Variant. Am J Pathol. 2003;163:2009-2019.

32. Jin R, Bay BH, Chow VT et al. Metallothionein 1F mRNA expression correlates with histological grade in breast carcinoma. Breast Cancer Res Treat. 2001;66:265-272.

33. Yap X, Tan HY, Huang $\mathbf{J}$ et al. Over-expression of metallothionein predicts chemoresistance in breast cancer.J Pathol. 2009;217:563-570.

34. Somji S, Sens MA, Lamm DL et al. Metallothionein isoform 1 and 2 gene expression in the human bladder: evidence for upregulation of MT-1X mRNA in bladder cancer. Cancer Detect Prev. 2001;25:62-75.

35. Garrett SH, Sens MA, Shukla D et al. Metallothionein isoform 1 and 2 gene expression in the human prostate: downregulation of MT-1X in advanced prostate cancer. Prostate. 2000;43:125-135. 